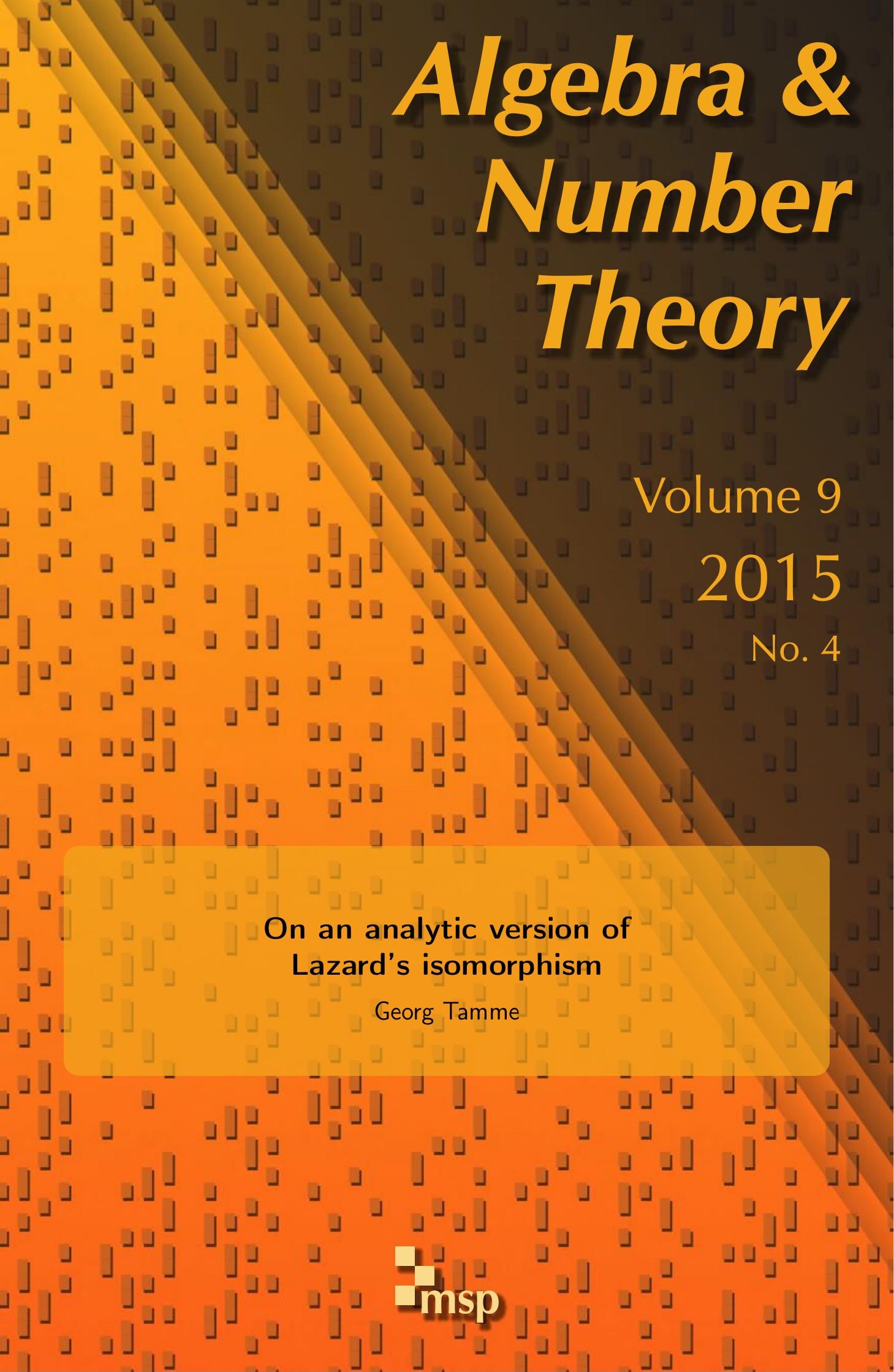




\title{
On an analytic version of Lazard's isomorphism
}

\author{
Georg Tamme
}

We prove a comparison theorem between locally analytic group cohomology and Lie algebra cohomology for locally analytic representations of a Lie group over a nonarchimedean field of characteristic 0. The proof is similar to that of van Est's isomorphism and uses only a minimum of functional analysis.

Introduction

1. Locally analytic group cohomology

2. Locally analytic representations

942

3. Differential forms and Lie algebra cohomology

945

4. Differential forms and locally analytic group cohomology

948

5. Explicit description of the comparison map

950

Appendix: The Poincaré lemma

952

References

955

\section{Introduction}

In a seminal paper, Lazard [1965] established two basic theorems concerning the cohomology of a compact $\mathbb{Q}_{p}$-analytic Lie group $G$ with Lie algebra $\mathfrak{g}$. Firstly, if $V$ is a finite-dimensional $\mathbb{Q}_{p}$-vector space with continuous $G$-action, the natural map from locally analytic group cohomology $H_{\mathrm{an}}^{*}(G, V)$, defined in terms of locally analytic cochains, to continuous group cohomology $H_{\text {cont }}^{*}(G, V)$ is an isomorphism. Secondly, there is a natural isomorphism between the direct limit $\operatorname{colim}_{G^{\prime} \subset G} H_{\text {cont }}^{*}\left(G^{\prime}, V\right)$, where $G^{\prime}$ runs through the system of open subgroups of $G$, and the Lie algebra cohomology $H^{*}(\mathfrak{g}, V)$. Hence, combining both, there is a natural isomorphism

$$
\underset{G^{\prime} \subset G \text { open }}{\operatorname{colim}} H_{\text {an }}^{*}\left(G^{\prime}, V\right) \cong H^{*}(\mathfrak{g}, V)
$$

The author is supported by the CRC 1085 Higher Invariants (Universität Regensburg). MSC2010: primary 22E50; secondary 17B56, 22E35.

Keywords: Lazard isomorphism, locally analytic group cohomology, Lia algebra cohomology. 
These results play an important role in arithmetic geometry, in particular in the theory of Galois representations, or in the study of $p$-adic regulators [Huber and Kings 2011].

At least for certain Lie groups, integral and $K$-analytic versions have been obtained by Huber, Kings, and Naumann [Huber et al. 2011] when $K$ is a finite extension of $\mathbb{Q}_{p}$. The proofs are based on Lazard's original argument via continuous group cohomology, and are not easily accessible. A somewhat simplified proof has been given by Lechner [2012] using formal group cohomology.

On the other hand, the situation for a real Lie group $G$ is much more transparent. The analogous result is van Est's isomorphism $H_{d}^{*}(G, V) \cong H^{*}(\mathfrak{g}, K ; V)$, which relates differentiable group cohomology with relative Lie algebra cohomology for a maximal compact subgroup $K \subseteq G$. Its proof is based on the following observations: The quotient $G / K$ is contractible, hence the de Rham complex $\Omega^{*}(G / K, V)$ with coefficients in a $G$-representation $V$ is a resolution of $V$. Moreover, for any $k$, the space $\Omega^{k}(G / K, V)$ is $G$-acyclic. Hence, $H_{d}^{*}(G, V)$ is computed by the $G$ invariants of the complex $\Omega^{*}(G / K, V)$, which is precisely the Chevalley-Eilenberg complex computing relative Lie algebra cohomology $H^{*}(\mathfrak{g}, K ; V)$.

It is a natural question whether a similar argument works in the nonarchimedean situation. In this note, we show that this is indeed the case. This gives a direct proof of the isomorphism (1) and generalizes it with respect to the ground field and the coefficients:

Theorem. Let $K$ be a nonarchimedean field of characteristic 0 . Let $G$ be a locally $K$-analytic Lie group and $G \rightarrow \operatorname{Aut}(V)$ a locally analytic representation on a barreled locally convex $K$-vector space. Denote by $\mathfrak{g}$ the $K$-Lie algebra of $G$. Then there are natural isomorphisms

$$
\underset{G^{\prime} \subset G \text { open }}{\operatorname{colim}} H_{\text {an }}^{*}\left(G^{\prime}, V\right) \cong H^{*}(\mathfrak{g}, V),
$$

where the colimit is taken over the system of open subgroups of $G$.

The rough argument is as follows: The de Rham complex $\Omega^{*}(G, V)$ is a resolution of the locally constant $V$-valued functions on $G$. As in the real case, each $\Omega^{k}(G, V)$ is $G$-acyclic, hence the cohomology of the locally constant $V$-valued functions on $G$ is isomorphic to the Lie algebra cohomology $H^{*}(\mathfrak{g}, V)$ (see Sections 3 and 4 for precise results). The theorem then follows by taking the direct limit over the open subgroups of $G$.

The proof also shows that, for compact $G$, one can recover the locally analytic group cohomology from the Lie algebra cohomology as the invariants under the natural $G$-action: $H_{\text {an }}^{*}(G, V) \cong H^{*}(\mathfrak{g}, V)^{G}$ (see Corollary 21$)$.

Moreover, we describe the comparison map between locally analytic group cohomology and Lie algebra cohomology explicitly on the level of complexes: It is 
given by differentiating locally analytic cocycles at 1 (see Section 5). As pointed out by the referee, one can use the methods of [Huber and Kings 2011] to show that, on cohomology groups, this comparison map agrees with the one studied by Lazard in the case that $K$ is $\mathbb{Q}_{p}$ and $V$ is finite-dimensional.

In order to apply usual arguments from homological algebra, we show, following [Flach 2008], that the locally analytic cochain cohomology groups can be interpreted as derived functors of the global section functor on a topos $B G$ (Sections 1 and 2). The nice feature of this is that it gives a quick proof of the main results which requires only a minimum of functional analysis.

An alternative approach to the cohomology of locally analytic representations of Lie groups over finite extensions of $\mathbb{Q}_{p}$ is due to Kohlhaase [2011]. It is based on relative homological algebra. He obtains similar results under an additional assumption on the group, which, as he proves, is fulfilled in many cases. The cohomology groups he defines are finer than ours in the sense that they themselves carry a locally convex topology. In contrast to the groups we use, they do not always coincide with the cohomology groups defined in terms of locally analytic cochains.

I would like to thank the referee for useful remarks, in particular concerning the comparison of our isomorphism with Lazard's original one.

Notations and conventions. For the whole paper, we let $K$ be a nonarchimedean field of characteristic 0 , i.e., $K$ is equipped with a nontrivial nonarchimedean absolute value $|\cdot|$ such that $K$ is complete for the topology defined by $|\cdot|$. By a manifold we will always mean a paracompact, finite-dimensional locally $K$-analytic manifold. Note that, by [Schneider 2011, Corollary 18.8], any locally $K$-analytic Lie group is paracompact. For manifolds $X, Y$, we denote by $C^{\text {an }}(X, Y)$ the set of locally $K$-analytic maps from $X$ to $Y$. We will refer to them simply as analytic maps.

\section{Locally analytic group cohomology}

In this section, we describe the topos-theoretic approach to locally analytic group cohomology. We refer to [Flach 2008] for the case of continuous cohomology.

Denote by La the category of manifolds. We let $\mathrm{Sh}(\mathrm{La})$ be the category of sheaves on La with respect to the topology generated by open coverings. For this topology, every representable presheaf is a sheaf, hence we have the Yoneda embedding $y: \mathrm{La} \rightarrow \mathrm{Sh}(\mathrm{La})$.

Let $G$ be a Lie group. Then $y(G)$ is a group object in $\mathrm{Sh}(\mathrm{La})$. The category of sheaves with a $y(G)$-action is a topos [SGA $4{ }_{1} 1972$, Exposé IV, 2.4], called the classifying topos of $y(G)$. It will be denoted by $B G{ }^{1}$ We denote its global section functor by $\Gamma: B G \rightarrow \operatorname{Set}, \Gamma(\mathscr{F})=\operatorname{Hom}_{B G}(*, \mathscr{F})=\mathscr{F}(*)^{G}$. Similarly, if $X$ is an

\footnotetext{
${ }^{1}$ More precisely, we assume the existence of universes and only consider manifolds which are elements of a given universe $\mathcal{U}$. Then $\operatorname{Sh}(\mathrm{La})$ and $B G$ are $\mathscr{V}$-topoi for a universe $\mathscr{V}$ with $\mathscr{U} \in \mathscr{V}$.
} 
object of $B G$, we denote by $\Gamma(X,-)=\operatorname{Hom}_{B G}(X,-)$ the functor of sections over $X$. As usual, we define cohomology groups via the derived functors of the global section functor:

Definition 2. Let $\mathscr{A}$ be an abelian group object of $B G$. Then we set

$$
H^{i}(G, \mathscr{A}):=\left(R^{i} \Gamma\right)(\mathscr{A}) .
$$

Example 3. Let $V$ be a finite-dimensional $K$-vector space with a linear $G$-action such that the map $G \times V \rightarrow V$ defining the action is analytic. This induces an action $y(G) \times y(V) \rightarrow y(V)$, and hence $y(V)$ can naturally be considered as an element of $B G$. We have $\Gamma(y(V))=V^{G}$. In the next section, we will show that higher cohomology groups $H^{i}(G, y(V))$ coincide with the cohomology groups defined in terms of locally analytic cochains with coefficients in $V$.

As another example, let $M$ be a continuous $G$-module, i.e., a topological abelian group equipped with a linear $G$-action such that $G \times M \rightarrow M$ is continuous. Then we have the sheaf of continuous $M$-valued functions $C(-, M)$ on La. It also carries a natural action by $y(G)$. It follows from Proposition 8 below that the groups $H^{i}(G, C(-, M))$ can be identified with the continuous cochain cohomology groups of $M$.

We want to describe the cohomology groups defined in Definition 2 in terms of a concrete complex. We begin with some general considerations.

Let $\mathscr{T}$ be a topos, and let $\mathscr{G}$ be a group object in $\mathscr{T}$. For objects $\mathscr{A}, \mathscr{B}$ of $B \mathscr{G}$ the internal hom $\mathscr{H} \operatorname{om}_{B} \mathscr{G}(\mathscr{A}, \mathscr{B})$ is given as follows: The underlying object of $\mathscr{T}$ is $\mathscr{H}_{o m_{\mathscr{T}}}(\mathscr{A}, \mathscr{B})$ and the action of $\mathscr{G}$ is given by the formula

$$
(g \phi)(a)=g\left(\phi\left(g^{-1} a\right)\right) .
$$

Denote by $i: * \rightarrow \mathscr{G}$ the morphism from the trivial group in $\mathscr{T}$ to $\mathscr{G}$. It induces a geometric morphism of topoi (see [SGA 41 1972, Exposé IV, 4.5])

$$
i: \mathscr{T} \cong B * \rightarrow B \mathscr{G} .
$$

The left adjoint $i^{*}$ simply forgets the $\mathscr{G}$-action. The right adjoint is given by $\left.i_{*}(\mathscr{F})=\mathscr{H}_{o m_{B} \mathscr{G}(l} \mathscr{G}, \mathscr{F}\right)$, where $l_{l} \mathscr{G}$ is $\mathscr{G}$ with its natural left action, viewed as an object ob $B \mathscr{G}$, and $\mathscr{F}$ is viewed as object of $B \mathscr{G}$ with trivial $\mathscr{G}$-action. The functor

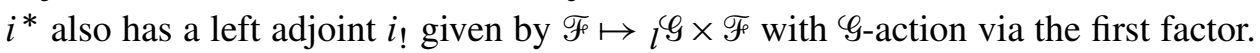

For an object $\mathscr{A} \in B_{\mathscr{G}}$, we denote by $\mathscr{A}^{\natural}$ the object of $B^{\mathscr{G}}$ with the same underlying object in $\mathscr{T}$ and trivial $\mathscr{G}$-action.

Lemma 4. For $\mathscr{A}, \mathscr{B} \in B \mathscr{G}$ we have

$$
\operatorname{Hom}_{B}\left(l(\mathcal{G} \times \mathscr{A}, \mathscr{B}) \cong i_{*} \mathscr{H o m}_{\mathscr{T}}\left(i^{*} \mathscr{A}, i^{*} \mathscr{B}\right) .\right.
$$


Proof. Let $\mathscr{X}$ be an object of $B \mathscr{G}$. Then we have natural isomorphisms

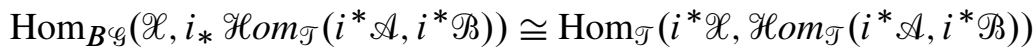

$$
\begin{aligned}
& \cong \operatorname{Hom}_{\mathscr{T}}\left(i^{*}(\mathscr{X} \times \mathscr{A}), i^{*} \mathscr{B}\right) \\
& \cong \operatorname{Hom}_{B} \mathscr{G}\left(i_{!} i^{*}(\mathscr{L} \times \mathscr{A}), \mathscr{B}\right) \\
& \cong \operatorname{Hom}_{B} \mathscr{G}\left(l_{l} \mathscr{G}(\mathscr{X} \times \mathscr{A})^{\natural}, \mathscr{B}\right) \\
& \cong \operatorname{Hom}_{B} \varphi(l \mathscr{G} \times \mathscr{X} \times \mathscr{A}, \mathscr{B}) \\
& \cong \operatorname{Hom}_{B} \mathscr{G}\left(\mathscr{X}, \mathscr{H}^{\circ} \operatorname{om}_{B}\left({ }_{l} \mathscr{G} \times \mathscr{A}, \mathscr{B}\right)\right),
\end{aligned}
$$

where we used the isomorphism $l_{l} \mathscr{G}(\mathscr{X} \times \mathscr{A})^{\natural} \cong l_{l} \mathscr{G} \mathscr{X} \times \mathscr{A}$ given by $\left(\mathrm{pr}_{1}\right.$, action). This implies the lemma.

We now consider the case $\mathscr{T}=\operatorname{Sh}(\mathrm{La}), \mathscr{G}=y(G)$. For a sheaf $\mathscr{F}$ on $\mathrm{La}$, the sheaf underlying $i_{*} \mathscr{F}$ is, by the above, given by $X \mapsto \mathscr{H}_{\operatorname{Som}(\mathrm{La})}(y(G), \mathscr{F})(X) \cong$ $\mathscr{F}(G \times X)$ (Yoneda lemma).

Remark 5. By our general assumption, every manifold $X$ in La is paracompact. By [Schneider 2011, Proposition 8.7], it is even strictly paracompact, i.e., every open covering of $X$ can be refined by a covering by pairwise-disjoint open subsets. This implies in particular that the functor of sections over $X$ is exact on the category of abelian sheaves on La.

Lemma 6. The functor $i_{*}$ from abelian sheaves on La to abelian group objects in $B G$ is exact.

Proof. Since $i_{*}$ is a right adjoint, it is left exact. Consider an epimorphism $\mathscr{A} \rightarrow \mathscr{B}$ of abelian sheaves on La. By the above remark, the functor of sections over $G \times X$ is exact, and hence $\mathscr{A}(G \times X) \rightarrow \mathscr{B}(G \times X)$ is an epimorphism of abelian groups. From this we deduce that $i_{*} \mathscr{A} \rightarrow i_{*} \mathscr{B}$ is an epimorphism.

Corollary 7. For any abelian sheaf $\mathscr{A}$ on $\mathrm{La}$, we have

$$
H^{i}\left(G, i_{*} \mathscr{A}\right) \cong \begin{cases}\mathscr{A}(*) & \text { if } i=0, \\ 0 & \text { else. }\end{cases}
$$

Proof. Since the left adjoint $i^{*}$ is exact, $i_{*}$ sends injectives to injectives. Since $i_{*}$ is exact and $\Gamma \circ i_{*}(-)=\operatorname{Hom}_{B G}\left(*, i_{*}(-)\right) \cong \operatorname{Hom}_{\mathrm{Sh}(\mathrm{La})}(*,-)$ we see that

$$
H^{i}\left(G, i_{*} \mathscr{A}\right) \cong H_{\mathrm{Sh}(\mathrm{La})}^{i}(*, \mathscr{A}) \cong \begin{cases}\mathscr{A}(*) & \text { if } i=0 \\ 0 & \text { else. }\end{cases}
$$

We let $E_{\bullet} G$ be the simplicial manifold given in degree $p$ by $E_{p} G:=G^{p+1}$, and $\phi^{*}: E_{q} G \rightarrow E_{p} G$, for $\phi:\{0<\cdots<p\} \rightarrow\{0<\cdots<q\}$, given by

$$
\left(g_{0}, \ldots, g_{q}\right) \mapsto\left(g_{\phi(0)}, \ldots, g_{\phi(p)}\right) .
$$


The group $G$ acts on $E_{\bullet} G$ via diagonal left multiplication. We denote the simplicial object of $B G$ given by $y\left(E_{\bullet} G\right)$ equipped with diagonal $y(G)$-action by ${ }_{l} E_{\bullet} G$.

For an abelian group object $\mathscr{A}$ in $B G$, the degree-wise sections over ${ }_{l} E_{\bullet} G$ form a cosimplicial abelian group $\Gamma\left({ }_{l} E_{\bullet} G, \mathscr{A}\right)$.

Proposition 8. Let $\mathscr{A}$ be an abelian group object of $B G$. Then

$$
H^{*}(G, \mathscr{A}) \cong H^{*}\left(\Gamma\left({ }_{l} E_{\bullet} G, \mathscr{A}\right)\right) .
$$

Proof. The projection $l y(G) \rightarrow *$ is an epimorphism in $B G$. The Čech nerve of this morphism is precisely ${ }_{l} E_{\bullet} G$. We thus have a quasi-isomorphism

$$
\mathscr{A} \cong \operatorname{Hom}_{B G}(*, \mathscr{A}) \stackrel{\sim}{\longrightarrow} \operatorname{Hom}_{B G}\left({ }_{l} E \cdot G, \mathscr{A}\right) .
$$

Using Lemma 4 and Corollary 7 we see that the complex on the right-hand side consists of $\Gamma$-acyclic objects. We conclude using $\Gamma\left(\mathscr{H}_{o m_{B G}}\left({ }_{l} E_{\bullet} G, \mathscr{A}\right)\right)=$ $\operatorname{Hom}_{B G}\left(*, \operatorname{Hom}_{B G}\left({ }_{l} E \cdot G, \mathscr{A}\right)\right) \cong \operatorname{Hom}_{B G}\left({ }_{l} E \bullet G, \mathscr{A}\right)=\Gamma\left({ }_{l} E_{\bullet} G, \mathscr{A}\right)$.

\section{Locally analytic representations}

In Example 3, we saw how to associate an abelian group object of $B G$ to any finite-dimensional locally analytic representation of $G$. The goal of this section is to extend this to arbitrary locally analytic representations in the sense of [Schneider and Teitelbaum 2002], and to relate the cohomology groups thus defined to the cohomology groups defined in terms of analytic cochains (Proposition 12).

We begin by recalling some basic notions about analytic functions and locally analytic representations. References are [Féaux de Lacroix 1998] or [Schneider and Teitelbaum 2002, §§2,3].

If $W$ is a Banach space, a function $f: X \rightarrow W$ from a manifold $X$ to $W$ is called analytic, if, in local charts, it is given by convergent power series with coefficients in $W$. The reader who is only interested in representations on Banach spaces can skip the following general definition and also all "admissibility" considerations later on. Let $V$ be a locally convex separated $K$-vector space. A $B H$-space ${ }^{2}$ for $V$ is a continuous inclusion of a separated Banach space $W \hookrightarrow V$ [Féaux de Lacroix $1998, \S 1.2]$. Let $X$ be a manifold. A function $f: X \rightarrow V$ is called analytic if every $x \in X$ admits a neighborhood $U$ and a BH-space $W \hookrightarrow V$ such that $\left.f\right|_{U}$ factors through an analytic map $U \rightarrow W$. We denote the set of all analytic functions $X \rightarrow V$ by $C^{\text {an }}(X, V)$. By [Féaux de Lacroix 1998, Korollar 2.4.4], $C^{\text {an }}(X, V)$ is a module over the algebra of analytic functions $C^{\text {an }}(X, K)$. For varying $X$, this is a sheaf on La denoted by $C^{\text {an }}(-, V)$.

\footnotetext{
${ }^{2}$ Banach-Hausdorff
} 
A topological representation of the Lie group $G$ on $V$ is an action of $G$ on $V$ by continuous automorphisms, i.e., a homomorphism $G \rightarrow \operatorname{Aut}(V)$ to the abstract group of continuous automorphisms $\operatorname{Aut}(V)$ of $V$. The topological representation is called locally analytic if all orbit maps $G \rightarrow V, g \mapsto g v$, are analytic (this is called a weakly analytic representation in [Féaux de Lacroix 1998, Definition 3.1.5]).

Example 9. Let $W$ be a Banach space. Then $\operatorname{Aut}(W)$ is an open subset of the Banach space of continuous endomorphisms End( $W)$. Féaux de Lacroix [1998, Korollar 3.1.9] showed that a topological representation of $G$ on $W$ is locally analytic if and only if the corresponding homomorphism $r: G \rightarrow \operatorname{Aut}(W) \subset \operatorname{End}(W)$ is analytic. Assume that this is the case. Let $X$ be a manifold, and let $\rho: X \rightarrow G$ and $f: X \rightarrow W$ be analytic maps. Then the point-wise product $\rho f: X \rightarrow W$, $x \mapsto \rho(x) f(x)$, is again analytic. Indeed, $\rho f$ equals the composition

$$
X \stackrel{\left(\rho, \mathrm{id}_{X}\right)}{\longrightarrow} G \times X \stackrel{r \times f}{\longrightarrow} \operatorname{Aut}(W) \times W \subseteq \operatorname{End}(W) \times W \stackrel{\mathrm{ev}}{\longrightarrow} W,
$$

where the first two maps are analytic by assumption and the evaluation map ev is continuous and bilinear. It follows that we get an action of $y(G)$ on the sheaf $C^{\text {an }}(-, W)$, and $C^{\text {an }}(-, W)$ can naturally be viewed as an object of $B G$. This generalizes Example 3 .

For a general locally analytic representation of $G$ on $V$, this need no longer be true. Instead, we have to consider the subsheaf of $C^{\text {an }}(-, V)$ of admissible functions, as we explain in the next paragraph. The example above shows that for representations on Banach spaces every analytic function is admissible.

Let $G \rightarrow \operatorname{Aut}(V)$ be a topological representation. We call an analytic function $f: X \rightarrow V$ admissible if the map $\hat{f}: G \times X \rightarrow V,(g, x) \mapsto g f(x)$ is analytic. Note that $\hat{f}$ is analytic if and only if its restriction $\left.\hat{f}\right|_{U \times X}$ for some open subset $U \subset G$ is analytic. Indeed, if this is the case, then for any $h \in G$ the restriction $\left.\hat{f}\right|_{h U \times X}$ is equal to the composition

$$
(h U \times X) \stackrel{(g, x) \mapsto\left(h^{-1} g, x\right)}{\longrightarrow} U \times X \stackrel{\left.\hat{f}\right|_{U \times X}}{\longrightarrow} V \stackrel{h \cdot}{\longrightarrow} V,
$$

where the first two maps are analytic and the last is continuous and linear. We define

$$
\underline{V}(X):=C^{\text {ad }}(X, V):=\left\{f \in C^{\text {an }}(X, V) \mid f \text { is admissible }\right\} .
$$

This is a $C^{\text {an }}(X, K)$-submodule of $C^{\text {an }}(X, V)$. We claim that $\underline{V}$ is a subsheaf of $C^{\text {an }}(-, V)$ and that the point-wise multiplication by $G$-valued analytic maps defines an action of $y(G)$ on $\underline{V}$. We will henceforth view $\underline{V}$ as an abelian group object of $B G$. 
Proof of the claim. If $f \in \underline{V}(X)$ and $\phi: Y \rightarrow X$ is an analytic map between manifolds, then $f \circ \phi$ is analytic. Moreover, $\widehat{f \circ \phi}=\hat{f} \circ\left(\operatorname{id}_{G} \times \phi\right)$ is analytic as well, hence $f \circ \phi$ is admissible. Thus $\underline{V}$ is a presheaf. Since admissibility is a local property, $\underline{V}$ is a sheaf.

Now let $\rho: X \rightarrow G$ be an analytic map. We define $\rho f$ by $(\rho f)(x):=\rho(x) f(x)$. We have to show that $\rho f$ is analytic and admissible. But this is clear since $\rho f$ equals the composition

$$
X \stackrel{\left(\rho, \operatorname{id}_{X}\right)}{\longrightarrow} G \times X \stackrel{\hat{f}}{\longrightarrow} V
$$

and $\widehat{\rho f}$ equals the composition

$$
G \times X \stackrel{(g, x) \mapsto(g \rho(x), x)}{\longrightarrow} G \times X \stackrel{\hat{f}}{\longrightarrow} V .
$$

Example 10. A topological representation of $G$ on $V$ is locally analytic if and only if $\underline{V}(*)=V$.

Definition 11. For a locally analytic representation of $G$ on $V$ and $i \geq 0$ we define the locally analytic group cohomology of $G$ with coefficients in $V$ as

$$
H_{\mathrm{an}}^{i}(G, V):=H^{i}(G, \underline{V}) .
$$

Recall that a homogeneous analytic $p$-cochain of $G$ with coefficients in $V$ is an analytic function $f: E_{p} G \rightarrow V$ which is $G$-equivariant, i.e., satisfies $f\left(g g_{0}, \ldots, g g_{p}\right)=g f\left(g_{0}, \ldots g_{p}\right)$. We denote the complex of homogeneous analytic cochains by $C_{G}^{\text {an }}\left(E_{\bullet} G, V\right)$. Its differential is induced by the simplicial structure of $E_{\bullet} G$.

Proposition 12. The cohomology $H_{\mathrm{an}}^{*}(G, V)$ is isomorphic to the cohomology of the complex of homogeneous analytic cochains $C_{G}^{\mathrm{an}}\left(E_{\bullet} G, V\right)$.

Proof. By Proposition 8 we have $H^{*}(G, \underline{V}) \cong H^{*}\left(\Gamma\left({ }_{l} E \bullet G, \underline{V}\right)\right)$. Using the Yoneda lemma we see that a section in $\Gamma\left({ }_{l} E_{p} G, \underline{V}\right)=\operatorname{Hom}_{B G}\left({ }_{l} E_{p} G, \underline{V}\right)$ is just an admissible function $f: E_{p} G \rightarrow V$ such that

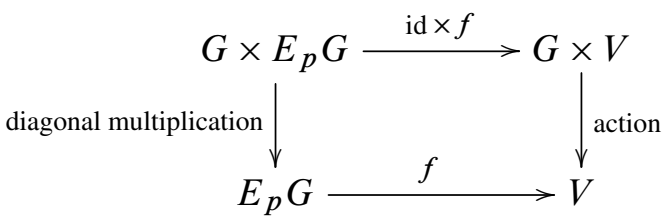

commutes, i.e., a $G$-equivariant admissible function $E_{p} G \rightarrow V$. 
To prove the claim, it suffices to show that, vice versa, every $G$-equivariant analytic function $f: E_{p} G \rightarrow V$ is admissible. But, by the $G$-equivariance, $\hat{f}$ is equal to the composition

$$
G \times E_{p} G \stackrel{\left(g,\left(g_{0}, \ldots, g_{p}\right)\right) \mapsto\left(g g_{0}, \ldots, g g_{p}\right)}{\longrightarrow} E_{p} G \stackrel{f}{\longrightarrow} V
$$

and thus analytic. Thus $f$ is admissible.

\section{Differential forms and Lie algebra cohomology}

In this section, we introduce sheaves of differential forms with coefficients in a locally analytic representation $V$ of $G$ as objects of $B G$. Again, unless $V$ is a Banach space, we have to restrict to admissible forms. We show that the Lie algebra cohomology of the $K$-Lie algebra $\mathfrak{g}$ of $G$ with coefficients in $V$ can be computed as the cohomology in $B G$ of the complex of forms on $G$ with coefficients in $V$.

Let $V$ be a separated locally convex $K$-vector space. For a submersion $Y \rightarrow X$ we denote by $\Omega^{k}(Y / X, V)$ the vector space of relative analytic $k$-forms with values in $V$. Here, a $k$-form $\omega$ is called analytic if for any $k$-tuple $\phi_{1}, \ldots, \phi_{k}$ of local sections of the vertical tangent bundle $T(Y / X)$ the function $Y \rightarrow V$, $y \mapsto \omega(y)\left(\phi_{1}(y), \ldots, \phi_{k}(y)\right)$ is analytic. It suffices to check this for the local sections given by a local frame of $T(Y / X)$. In particular, every $y \in Y$ admits a neighborhood $U$ and a BH-space $W \hookrightarrow V$ such that $\left.\omega\right|_{U}$ is in the image of $\Omega^{k}(U / X, W) \hookrightarrow \Omega^{k}(U / X, V)$. It follows that the exterior derivative $d \omega$ is a welldefined form in $\Omega^{k+1}(Y / X, V)$. If $V$ is finite-dimensional, this is the usual notion of analytic forms.

For a fixed manifold $Y$, we have a complex of sheaves $\underline{\Omega}^{*}(Y, V)$ on La defined by

$$
\underline{\Omega}^{*}(Y, V)(X):=\Omega^{*}(X \times Y / X, V) .
$$

Let $V$ be a locally analytic representation of $G$. We would like to equip this complex with a $y(G)$-action. As for functions, we have to restrict to a subcomplex of admissible forms in order to do this. Again, one can ignore this, if one is only interested in the case that $V$ is a Banach space.

A form $\omega \in \Omega^{k}(Y / X, V)$ is called admissible if the form $\hat{\omega}$ on $G \times Y / G \times X$ given by

$$
\hat{\omega}(g, y)\left(\mathrm{x}_{1}, \ldots, \mathrm{x}_{k}\right):=g \cdot\left(\omega(y)\left(\mathrm{x}_{1}, \ldots, \mathrm{x}_{k}\right)\right),
$$

where $g \in G, y \in Y$ and $x_{i} \in T_{(g, y)}(G \times Y / G \times X) \cong T_{y}(Y / X)$, is analytic. Equivalently, $\omega$ is admissible if and only if for any $k$-tuple of local sections $\phi_{1}, \ldots, \phi_{k}$ of $T(Y / K)$ as above the function $\omega\left(\phi_{1}, \ldots, \phi_{k}\right)$ is admissible. As in the case of functions, this is the case if and only if $\left.\hat{\omega}\right|_{U \times Y}$ is analytic for some open subset $U \subseteq G$. The admissible $k$-forms form a $C^{\text {an }}(Y, K)$-submodule of $\Omega^{k}(Y / X, V)$, which we denote by $\Omega_{\mathrm{ad}}^{k}(Y / X, V)$. They are also stable under 
the exterior derivative: Let $\omega$ be an admissible form. Since $G$ acts on $V$ by continuous linear automorphisms we have $\widehat{d \omega}=d \hat{\omega}$, and this form is analytic. Thus, the admissible forms $\Omega_{\mathrm{ad}}^{*}(Y / X, V)$ form a subcomplex of the de Rham complex $\Omega^{*}(Y / X, V)$.

Example 13. If $V$ is a Banach space, it follows from Example 9 that any $V$-valued analytic $k$-form is admissible.

We now fix a manifold $Y$. For an analytic map between manifolds $X^{\prime} \rightarrow X$, the pullback map $\Omega^{k}(X \times Y / X) \rightarrow \Omega^{k}\left(X^{\prime} \times Y / X^{\prime}\right)$ preserves admissible forms. Since admissibility is a local condition, $\underline{\Omega}_{\mathrm{ad}}^{k}(Y, V)$, defined by

$$
\underline{\Omega}_{\mathrm{ad}}^{k}(Y, V)(X):=\Omega_{\mathrm{ad}}^{k}(X \times Y / X, V),
$$

is a subsheaf of $\underline{\Omega}^{k}(Y, V)$, and $\underline{\Omega}_{\text {ad }}^{*}(Y, V) \subseteq \underline{\Omega}^{*}(Y, V)$ is a subcomplex.

We define an action of $y(G)$ on $\underline{\Omega}_{\mathrm{ad}}^{k}(G, V)$ as follows: Let $\rho: X \rightarrow G$ be an analytic map and $\omega \in \Omega_{\mathrm{ad}}^{k}(X \times G / X, V)$ an admissible form. For any $h \in G$, left translation by $h^{-1}$ induces a map $T_{(x, g)}(X \times G / X) \rightarrow T_{\left(x, h^{-1} g\right)}(X \times G / X)$, written $\mathrm{x} \mapsto h^{-1} \times$. Using this notation, we define $\rho \omega$ by the formula

$$
(\rho \omega)(x, g)\left(\mathrm{x}_{1}, \ldots, \mathrm{x}_{k}\right):=\rho(x) \cdot\left(\omega\left(x, \rho(x)^{-1} g\right)\left(\rho(x)^{-1} \mathrm{x}_{1}, \ldots, \rho(x)^{-1} \mathrm{x}_{k}\right)\right) .
$$

Lemma 14. This is a well-defined $y(G)$-action.

Proof. We have to show that $\rho \omega$ is analytic and admissible. Consider the analytic maps $\hat{\rho}: G \times X \times G \rightarrow G \times X \times G,(h, x, g) \mapsto\left(h \rho(x), x, \rho(x)^{-1} g\right)$ and $i_{1}$ : $X \times G \hookrightarrow G \times X \times G,(x, g) \mapsto(1, x, g)$. Then $\rho \omega=i_{1}^{*} \hat{\rho}^{*} \hat{\omega}$; hence $\rho \omega$ is analytic. Similarly, we have $\widehat{\rho \omega}=\hat{\rho}^{*} \hat{\omega}$, and hence $\rho \omega$ is admissible.

We thus consider $\underline{\Omega}_{\mathrm{ad}}^{k}(G, V)$ as an abelian group object in $B G$. We want to show that it is acyclic. Write $\widetilde{V}:=\operatorname{Hom}\left(\bigwedge^{k} \mathfrak{g}, V\right)$. The adjoint action of $G$ on $\mathfrak{g}$ and the given action of $G$ on $V$ induce a natural $G$-action on $\tilde{V}$.

Lemma 15. This representation of $G$ on $\tilde{V}$ is locally analytic. We have an isomorphism

$$
\underline{\Omega}_{\mathrm{ad}}^{k}(G, V) \cong \operatorname{Hom}_{B G}(l y(G), \underline{\tilde{V}}) \cong i_{*} i^{*} \underline{\tilde{V}} .
$$

Proof. Let $Y$ be a manifold. We claim that a function $f: Y \rightarrow \widetilde{V}$ is admissible if and only if the function $f_{\mathrm{x}}: Y \rightarrow V, y \mapsto f(y)(\mathrm{x})$ is admissible for every $\mathrm{x} \in \wedge^{k} \mathfrak{g}$. Taking $Y$ to be a point this implies the first assertion of the lemma.

To prove the claim, assume first that $f$ is admissible. We have $\widehat{f}_{\mathrm{x}}(g, y)=$ $g(f(y)(\mathrm{x}))=(g f(y))(g \times)=\hat{f}(g, y)(g \times)$. The function $\hat{f}$ is analytic by assumption and so is $g \mapsto g \times$. Since the evaluation $\widetilde{V} \times \wedge^{k} \mathfrak{g} \rightarrow V$ is continuous and bilinear, and since $\wedge^{k} \mathfrak{g}$ is finite dimensional, [Féaux de Lacroix 1998, Satz 2.4.3] implies that $\widehat{f}_{\mathrm{x}}$ is analytic. 
To see the converse, let $\mathrm{x}_{1}, \ldots, \mathrm{x}_{N}$ be a basis of $\bigwedge^{k} \mathfrak{g}$ and $\mathrm{x}_{1}^{*}, \ldots, \mathrm{x}_{N}^{*}$ the dual basis of $\left(\bigwedge^{k} \mathfrak{g}\right)^{\vee}$. We can write $f$ as a sum $f=\sum_{i=1}^{N} f_{\mathrm{x}_{i}} \mathrm{x}_{i}^{*}$ with $f_{\mathrm{x}_{i}}$ admissible. Then $\hat{f}(g, y)=\sum_{i} \widehat{\mathrm{f}_{i}}(g, y) g\left(\mathrm{x}_{i}^{*}\right)$ and, by [Féaux de Lacroix 1998, Satz 2.4.3] again, $\hat{f}$ is analytic.

We now prove the second assertion of the lemma. For any manifold $X$, right translations by elements of $G$ induce a trivialization of the vertical tangent bundle $T(X \times G / X) \cong(X \times G) \times \mathfrak{g}$. This gives a natural isomorphism of vector spaces

$$
\Omega^{k}(X \times G / X, V) \cong C^{\text {an }}(X \times G, \tilde{V}) .
$$

Using the above claim one sees that this isomorphism restricts to an isomorphism

$$
\Omega_{\mathrm{ad}}^{k}(X \times G / X, V) \cong C^{\mathrm{ad}}(X \times G, \tilde{V}) .
$$

Under this isomorphism, the $y(G)(X)$-action on the left-hand side corresponds to the action on the right-hand side induced by left translations on $X \times G$ and the action on $\widetilde{V}$ mentioned above. Using the isomorphism $C^{\text {ad }}(X \times G, \tilde{V}) \cong$ $\operatorname{Hom}_{B G}(l y(G), \underline{\tilde{V}})(X)$, this gives the first isomorphism stated in the Lemma. The second follows immediately from Lemma 4.

Corollary 16. We have

$$
H^{i}\left(G, \underline{\Omega}_{\mathrm{ad}}^{k}(G, V)\right) \cong \begin{cases}\operatorname{Hom}_{K}\left(\bigwedge^{k} \mathfrak{g}, V\right) & \text { if } i=0, \\ 0 & \text { else. }\end{cases}
$$

Proof. By Lemma 15 and Corollary 7 the higher cohomology groups vanish, and

$$
H^{0}\left(G, \underline{\Omega}_{\mathrm{ad}}^{k}(G, V)\right) \cong \underline{\tilde{V}}(*)=\operatorname{Hom}_{K}\left(\bigwedge^{k} \mathfrak{g}, V\right),
$$

concluding the proof.

Explicitly, this isomorphism is given by evaluating a form at $1 \in G$.

The differential $d$ of the complex $\underline{\Omega}_{\text {ad }}^{*}(G, V)$ is compatible with the $y(G)$-action. Hence we can view $\underline{\Omega}_{\mathrm{ad}}^{*}(G, V)$ as a complex in $B G$ and we can compute its hypercohomology.

We now assume that $V$ is barreled, i.e., that every closed convex absorbing subset is open (see [Schneider 2002, §6]). For example, any complete metrizable locally convex space, in particular any Banach space, is barreled. Differentiating the orbit maps $g \mapsto g v$ then induces an action of the Lie algebra $\mathfrak{g}$ on $V$ [Féaux de Lacroix 1998, Sätze 3.1.3, 3.1.7].

Corollary 18. We have natural isomorphisms

$$
H^{i}\left(G, \underline{\Omega}_{\mathrm{ad}}^{*}(G, V)\right) \cong H^{i}(\mathfrak{g}, V),
$$

where the right-hand side is Lie algebra cohomology. 
Proof. Corollary 16 gives an isomorphism

$$
H^{i}\left(G, \underline{\Omega}_{\mathrm{ad}}^{*}(G, V)\right) \cong H^{i}\left(\operatorname{Hom}_{K}\left(\bigwedge^{*} \mathfrak{g}, V\right)\right),
$$

where the differential on $\operatorname{Hom}_{K}\left(\bigwedge^{*} \mathfrak{g}, V\right)$ is induced from the de Rham differential via (17). This is precisely the Chevalley-Eilenberg complex computing Lie algebra cohomology.

\section{Differential forms and locally analytic group cohomology}

As before, we fix a locally analytic representation $G \rightarrow \operatorname{Aut}(V)$. In this section we use the Poincaré lemma to compare the hypercohomology of the complex of $V$-valued admissible forms with locally analytic group cohomology, and we give the proof of the theorem announced on page 938 in the introduction.

Fix a manifold $Y$. A function $f: Y \times X \rightarrow V$ will be called locally constant along $Y$ if, for every $(y, x) \in Y \times X$, there exist open neighborhoods $Y^{\prime} \subseteq Y$ of $y$ and $X^{\prime} \subseteq X$ of $x$ such that $\left.f\right|_{Y^{\prime} \times X^{\prime}}$ factors through the projection $Y^{\prime} \times X^{\prime} \rightarrow X^{\prime}$. We define

$$
\underline{C}_{\mathrm{ad}}^{\mathrm{lc}}(Y, V)(X):=\left\{f \in C^{\mathrm{ad}}(X \times Y, V) \mid f \text { is locally constant along } Y\right\} .
$$

It is easy to see that $X \mapsto \underline{C}_{\text {ad }}^{\text {lc }}(Y, V)(X)$ defines a sheaf on La.

Proposition 19. The inclusion in degree 0

$$
\underline{C}_{\mathrm{ad}}^{\mathrm{lc}}(Y, V) \rightarrow \underline{\Omega}_{\mathrm{ad}}^{*}(Y, V)
$$

is a quasi-isomorphism.

If $V$ is a Banach space, this is just the Poincaré lemma, and its usual proof works. For general locally convex $V$, it is a little bit more complicated, since we have to prove admissibility of primitives.

Proof. The map clearly induces an isomorphism on $H^{0}$, and it remains to show that $H^{k}\left(\underline{\Omega}_{\mathrm{ad}}^{*}(Y, V)\right)=0$ for $k>0$.

Let $X$ be a manifold, and let $\omega$ be a closed form in $\Omega_{\text {ad }}^{k}(X \times Y / X, V)$. We will show that there is an $\eta \in \Omega_{\text {ad }}^{k-1}(X \times Y / X, V)$ such that $d \eta=\omega$. Since all manifolds are strictly paracompact, it is enough to construct such an $\eta$ locally on $X$ and $Y$ (see Remark 5).

The rest of the proof uses some results and notations from the Appendix. It can be skipped on first reading. Since $d \hat{\omega}=\widehat{d \omega}=0$, the form $\hat{\omega} \in \Omega^{k}(G \times X \times Y / G \times X, V)$ is closed. Replacing $G$ be a small open neighborhood of 1 and using local charts, we may assume that there are multiradii $\delta \in \mathbb{R}_{+}^{m}, \in \in \mathbb{R}_{+}^{n}$ such that $G \times X \cong$ $B_{\delta}(0) \subset K^{m}, Y \cong B_{\epsilon}(0) \subset K^{n}$, and a BH-space $W \hookrightarrow V$ such that $\hat{\omega}$ is given by a power series in $F_{\delta}\left(\Omega_{\epsilon}^{k}(W)\right)$ (see (25)). Choose a multiradius $\epsilon^{\prime}<\epsilon$. The homotopy operator $h: \Omega_{\epsilon}^{k}(W) \rightarrow \Omega_{\epsilon^{\prime}}^{k-1}(W)$ given by Lemma 26 induces an operator 
$h: F_{\delta}\left(\Omega_{\epsilon}^{k}(W)\right) \rightarrow F_{\delta}\left(\Omega_{\epsilon^{\prime}}^{k-1}(W)\right)$. We set $\tilde{\eta}:=h(\hat{\omega})$. Hence $\tilde{\eta}$ represents a relative analytic $(k-1)$-form on $G \times X \times Y^{\prime} / G \times X$ with an open subset $Y^{\prime} \subset Y$. Since $\hat{\omega}$ is closed, we have $d \widetilde{\eta}=\left.\hat{\omega}\right|_{G \times X \times Y^{\prime}}$.

For $g \in G$, let $i_{g}: X \times Y \rightarrow G \times X \times Y$ (and similarly with $Y$ replaced by $Y^{\prime}$ ) be the inclusion $(x, y) \mapsto(g, x, y)$. We set $\eta:=i_{1}^{*} \widetilde{\eta}$. Clearly, $d \eta=i_{1}^{*} d \tilde{\eta}=i_{1}^{*} \hat{\omega}=\omega$. To prove that $\eta$ is admissible, we show that $\hat{\eta}=\widetilde{\eta}$. Let $\Phi_{g}: V \rightarrow V$ be the continuous automorphism given by the action of $g$. We have to check that $i_{g}^{*} \widetilde{\eta}=\Phi_{g} \circ \eta$. By restriction, $\Phi_{g}$ induces a continuous isomorphism of BH-spaces $W \rightarrow g(W)$ (more precisely, we view $W$ as a linear subspace of $V$ and let $g(W)$ be its image under the action of $g \in G$ with Banach space structure induced from $W$ via the linear isomorphism $\left.\Phi_{g}\right|_{W}: W \stackrel{\sim}{\longrightarrow} g(W)$ ). We have

$$
\begin{aligned}
i_{g}^{*} \tilde{\eta} & =i_{g}^{*}(h(\hat{\omega})) & & \text { (by definition) } \\
& =h\left(i_{g}^{*} \hat{\omega}\right) & & \left(\text { using (24) with } \Phi=h: \Omega_{\epsilon}^{q}(W) \rightarrow \Omega_{\epsilon^{\prime}}^{q-1}(W)\right) \\
& =h\left(\Phi_{g} \circ \omega\right) & & (\text { definition of } \hat{\omega}) \\
& =\Phi_{g} \circ h(\omega) & & \left(\text { Lemma } 27 \text { for } \Phi=\Phi_{g}: W \rightarrow g(W)\right) \\
& =\Phi_{g} \circ \eta & & \left(\text { since } h(\omega)=h\left(i_{1}^{*} \hat{\omega}\right)=i_{1}^{*} h(\hat{\omega})=i_{1}^{*} \hat{\eta}=\eta\right) .
\end{aligned}
$$

Proof of the Theorem. The sheaf $\underline{C}_{\mathrm{ad}}^{\mathrm{lc}}(G, V)$ carries a natural $y(G)$-action induced by left translations on $G$ and the given action on $V$. By Proposition 19 and Corollary 18 we have isomorphisms

$$
H^{*}\left(G, \underline{C}_{\mathrm{ad}}^{\mathrm{lc}}(G, V)\right) \cong H^{*}\left(G, \underline{\Omega}_{\mathrm{ad}}^{*}(G, V)\right) \cong H^{*}(\mathfrak{g}, V)
$$

As in the proof of Proposition 12, Proposition 8 implies that $H^{*}\left(G, \underline{C}_{\mathrm{ad}}^{\mathrm{lc}}(G, V)\right)$ is the cohomology of the complex $C_{G}^{\mathrm{lc}}\left(G \times E_{\bullet} G, V\right)$ of $G$-equivariant analytic functions $G \times E_{p} G \rightarrow V$ that are locally constant along the first factor.

Since the open subgroups $G^{\prime} \subseteq G$ form a fundamental system of neighborhoods of $1 \in G$ (see [Schneider 2011, Lemma 18.7]), we have an isomorphism

$$
\underset{G^{\prime} \subset G \text { open }}{\operatorname{colim}} C_{G^{\prime}}^{\mathrm{an}}\left(E_{\bullet} G^{\prime}, V\right) \cong \underset{G^{\prime} \subset G \text { open }}{\operatorname{colim}} C_{G^{\prime}}^{\mathrm{lc}}\left(G^{\prime} \times E_{\bullet} G^{\prime}, V\right) .
$$

Because taking the colimit over a directed system is exact, we see that

$$
\underset{G^{\prime} \subset G}{\operatorname{colim}} H_{\mathrm{an}}^{*}\left(G^{\prime}, V\right) \rightarrow \underset{G^{\prime} \subset G}{\operatorname{colim}} H^{*}\left(G^{\prime}, \underline{C}^{\mathrm{lc}}\left(G^{\prime}, V\right)\right)
$$

is an isomorphism. Since the isomorphisms (20) are compatible with the restriction to open subgroups, the claim follows. 
There is an additional action of $G$ on $\underline{C}_{\mathrm{ad}}^{\text {lc }}(G, V)$ and on $\underline{\Omega}_{\mathrm{ad}}^{*}(G, V)$ induced by right translations on $G$. This action is compatible with the given $y(G)$-action. It induces a $G$-action on the cohomology groups. Via the isomorphism (20) this corresponds to the $G$-action on $H^{*}(\mathfrak{g}, V)$ induced by the adjoint action on $\mathfrak{g}$ and left multiplication on $V$.

Corollary 21. If $G$ is compact, there is a natural isomorphism

$$
H_{\mathrm{an}}^{*}(G, V) \cong H^{*}(\mathfrak{g}, V)^{G} .
$$

Proof. Since $G$ is compact, every open subgroup is of finite index and contains an open normal subgroup. If $X$ is a compact manifold, every function in $\underline{C}_{\mathrm{ad}}^{\mathrm{lc}}(G, V)(X)$ factors through $G / H \times X$ for some open normal subgroup $H \unlhd G$. Thus - using the notation from the previous proof -

$$
\underline{C}_{G}^{\mathrm{lc}}\left(G \times E_{\bullet} G, V\right)=\underset{H \unlhd G \text { open }}{\operatorname{colim}} C_{G}^{\mathrm{an}}\left(G / H \times E_{\bullet} G, V\right) .
$$

Since the colimit over a directed system is exact, this induces an isomorphism $H^{*}(\mathfrak{g}, V) \cong \operatorname{colim}_{H \unlhd G} H^{*}\left(C_{G}^{\text {an }}\left(G / H \times E_{\bullet} G, V\right)\right)$. Since each quotient $G / H$ is finite, and taking invariants under a finite group is an exact functor on $K$-vector spaces with an action by that group, we get

$$
\begin{aligned}
H^{*}(\mathfrak{g}, V) & \cong \operatorname{colim}_{H \unlhd G} H^{*}\left(C_{G}^{\text {an }}\left(G / H \times E_{\bullet} G, V\right)\right)^{G / H} \\
& \cong \operatorname{colim}_{H \unlhd G} H^{*}\left(C_{G}^{\text {an }}\left(G / H \times E_{\bullet} G, V\right)^{G / H}\right) \\
& \cong \operatorname{colim}_{H \unlhd G} H^{*}\left(C_{G}^{\text {an }}(E \bullet G, V)\right) \cong H_{\text {an }}^{*}(G, V) .
\end{aligned}
$$

\section{Explicit description of the comparison map}

We want to describe an explicit map of complexes which induces the comparison map $H_{\text {an }}^{*}(G, V) \rightarrow H^{*}(\mathfrak{g}, V)$. Recall that $H_{\text {an }}^{*}(G, V)$ is computed by the complex of homogeneous locally analytic cochains $C_{G}^{\text {an }}(E \bullet G, V)$, and that $H^{*}(\mathfrak{g}, V)$ is computed by the complex of $G$-invariant admissible differential forms $\Omega_{\mathrm{ad}}^{*}(G, V)^{G}$.

For integers $p \geq 0$ and $0 \leq i \leq p$, we denote by $d_{i}$ the partial exterior derivative in the direction of the $(i+1)$-th factor of the product $E_{p} G=G^{p+1}$. We denote by $\Delta_{p}: G \rightarrow E_{p} G$ the diagonal map. For $f \in C^{\text {an }}\left(E_{p} G, V\right)$ we set

$$
\Psi(f):=\Delta_{p}^{*}\left(d_{1} d_{2} \ldots d_{p} f\right) \in \Omega^{p}(G, V) .
$$

Proposition 22. The map $\Psi$ induces a morphism of complexes $C_{G}^{\mathrm{an}}\left(E_{\bullet} G, V\right) \rightarrow$ $\Omega_{\mathrm{ad}}^{*}(G ; V)^{G}$, which agrees with the comparison map $H_{\mathrm{an}}^{*}(G, V) \rightarrow H^{*}(\mathfrak{g}, V)$ on cohomology groups. 
Remark 23. Let us consider the special case that $K$ is $\mathbb{Q}_{p}$ and $V$ is finite dimensional. We want to indicate how the method of [Huber and Kings 2011] allows one to compare our map with Lazard's one. The space of functions $C^{\text {an }}\left(E_{p} G, V\right)$ has topological generators of the form $f_{0} \otimes \cdots \otimes f_{p} \otimes v$ with $f_{i} \in C^{\text {an }}(G, K)$ and $v \in V$. For such a function we have

$$
\Psi\left(f_{0} \otimes \cdots \otimes f_{p} \otimes v\right)=f_{0} d f_{1} \wedge \cdots \wedge d f_{p} \otimes v
$$

and its image in $\operatorname{Hom}\left(\wedge^{p} \mathfrak{g}, V\right)$ is given by $f_{0}(1) d f_{1}(1) \wedge \cdots \wedge d f_{p}(1) \otimes v$.

There is another simplicial model $\widetilde{E}_{\bullet} G$ for the universal $G$-bundle (see [Huber and Kings $2011, \S 4.4]$ ), given by $\widetilde{E}_{p} G=E_{p} G$, but with face maps

$$
\tilde{\partial}_{i}\left(g_{0}, \ldots, g_{n}\right)= \begin{cases}\left(g_{0}, \ldots, g_{i-1}, g_{i} g_{i+1}, g_{i+2}, \ldots, g_{p}\right) & \text { if } i=0, \ldots p-1 \\ \left(g_{0}, \ldots, g_{p-1}\right) & \text { if } i=p\end{cases}
$$

The $G$-action on $\widetilde{E}_{\bullet} G$ is given by left multiplication on the first factor. There is a natural $G$-equivariant isomorphism $\widetilde{E}_{\bullet} G \cong E_{\bullet} G$. Huber and Kings show that Lazard's isomorphism (for $G$ small enough) is induced by the map

$$
\begin{aligned}
\Phi: C_{G}^{\mathrm{an}}\left(\widetilde{E}_{\bullet} G, V\right) & \rightarrow \operatorname{Hom}\left(\wedge^{\bullet} \mathfrak{g}, V\right), \\
\Phi\left(f_{0} \otimes \cdots \otimes f_{p} \otimes v\right) & =f_{0}(1) d f_{1}(1) \wedge \cdots \wedge d f_{p}(1) \otimes v
\end{aligned}
$$

(see [Huber and Kings 2011, Proposition 4.6.1]; this is formulated in the case of trivial coefficients, but can easily be adapted to our setting). The argument of [Huber and Kings 2011, Theorem 4.7.1] shows that the composition of $\Phi$ with the isomorphism $C_{G}^{\text {an }}\left(E_{\bullet} G, V\right) \cong C_{G}^{\text {an }}\left(E_{\bullet} G, V\right)$ is homotopic to $\Psi$, hence both maps agree on cohomology groups.

Proof of Proposition 22. From the proof of Proposition 8 we have the acyclic resolution $\underline{V} \stackrel{\sim}{\rightarrow} \operatorname{Hom}_{B G}\left({ }_{l} E_{\bullet} G, \underline{V}\right)$. For a manifold $X$ we have

$$
\operatorname{Hom}_{B G}\left({ }_{l} E_{\bullet} G, \underline{V}\right)(X)=C^{\mathrm{ad}}\left(X \times E_{\bullet} G, V\right)
$$

with $y(G)$-action induced from left translations on $E_{\bullet} G$ and the action on $V$. We define $\Psi: C^{\text {ad }}\left(X \times E_{p} G, V\right) \rightarrow \Omega_{\text {ad }}^{p}(X \times G / X, V)$ by the same formula as above. We claim that this gives a morphism of complexes $\Psi: \operatorname{Hom}_{B G}\left({ }_{l} E \bullet G, \underline{V}\right) \rightarrow \underline{\Omega}_{\mathrm{ad}}^{*}(G, V)$ in $B G$.

Proof of the claim. One checks without difficulty that $\Psi$ is equivariant for the $y(G)$-action. Now consider $f \in C^{\text {ad }}\left(E_{p} G \times X, V\right)$. Recall the face maps

$$
\partial_{i}: E_{p+1} G \rightarrow E_{p} G, \quad\left(g_{0}, \ldots, g_{p+1}\right) \mapsto\left(g_{0}, \ldots, \widehat{g_{i}}, \ldots, g_{p+1}\right) .
$$


The differential of the complex $C^{\text {ad }}\left(E_{\bullet} G \times X, V\right)$ maps $f$ to

$$
\sum_{i=0}^{p+1}(-1)^{i} \partial_{i}^{*} f
$$

Since $\partial_{i}^{*} f$ is constant along the $(i+1)$-th factor $G$, we have $d_{i}\left(\partial_{i}^{*} f\right)=0$. Since the partial derivatives commute up to sign, it follows that

$$
\begin{aligned}
\Psi\left(\sum_{i=0}^{p+1}(-1)^{i} \partial_{i}^{*} f\right) & =\Psi\left(\partial_{0}^{*} f\right) \\
& =\Delta_{p+1}^{*}\left(d_{1} \ldots d_{p+1}\left(\partial_{0}^{*} f\right)\right) \\
& =\Delta_{p+1}^{*}\left(\partial_{0}^{*}\left(d_{0} \ldots d_{p} f\right)\right) \\
& =\Delta_{p}^{*}\left(d_{0} \ldots d_{p} f\right) \\
& =\Delta_{p}^{*}\left(d\left(d_{1} \ldots d_{p} f\right)\right) \\
& =d\left(\Delta_{p}^{*}\left(d_{1} \ldots d_{p} f\right)\right) \\
& =d(\Psi(f)) .
\end{aligned}
$$

We thus have a commutative diagram

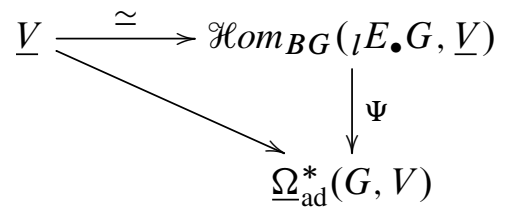

where the complexes on the right-hand side consist of acyclic sheaves. The proposition now follows by taking global sections.

\section{Appendix: The Poincaré lemma}

Let $W$ be a $K$-Banach space with norm $\|\cdot\|$. For a multiradius $\epsilon=\left(\epsilon_{1}, \ldots, \epsilon_{n}\right) \in \mathbb{R}_{+}^{n}$ we denote the space of $\epsilon$-convergent power series in $n$ variables $x=\left(x_{1}, \ldots, x_{n}\right)$ with coefficients in $W$ by $F_{\epsilon}(W)$ :

$$
F_{\epsilon}(W):=\left\{\sum_{I \in \mathbb{N}_{0}^{n}} a_{I} x^{I} \mid a_{I} \in W,\left\|a_{I}\right\| \epsilon^{I} \stackrel{I \rightarrow \infty}{\longrightarrow} 0\right\} .
$$

Equipped with the norm $\left\|\sum_{I} a_{I} x^{I}\right\|_{\epsilon}:=\max _{I}\left\|a_{I}\right\| \epsilon^{I}$, this is again a Banach space.

Let $\Phi: W \rightarrow W^{\prime}$ be a continuous linear map between Banach spaces. It induces a continuous linear map $F_{\epsilon}(W) \rightarrow F_{\epsilon}\left(W^{\prime}\right)$. Let $B_{\epsilon}(0) \subset K^{n}$ be the closed ball of radius $\epsilon$ around 0 . For any $x \in B_{\epsilon}(0)$ we have the evaluation at $x$, written 
$i_{x}^{*}: F_{\epsilon}(W) \rightarrow W$, and similarly for $W^{\prime}$. Since $\Phi$ is continuous the diagram

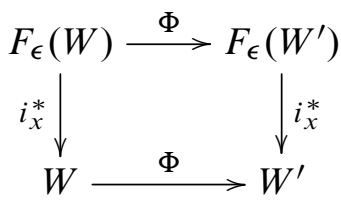

commutes.

For $q \geq 0$ we denote by $\Omega_{\epsilon}^{q}(W)$ the space of $\epsilon$-convergent $W$-valued $q$-forms in $n$ variables:

$$
\Omega_{\epsilon}^{q}(W):=\bigwedge_{K}^{q}\left(K^{n}\right)^{\vee} \otimes_{K} F_{\epsilon}(W) .
$$

Since $\bigwedge_{K}^{q}\left(K^{n}\right)^{\vee}$ is a finite-dimensional $K$-vector space, this is again a Banach space. The usual differential defines a continuous linear map $d: \Omega_{\epsilon}^{q}(W) \rightarrow \Omega_{\epsilon}^{q+1}(W)$.

There is a natural injection $\Omega_{\epsilon}^{q}(W) \hookrightarrow \Omega^{q}\left(B_{\epsilon}(0), W\right)$ into the space of locally analytic $W$-valued $q$-forms. It is compatible with the differential. More generally, if $\delta \in \mathbb{R}_{+}^{m}$ is a second multiradius, we can identify $\delta$-convergent power series with coefficients in $\Omega_{\epsilon}^{q}(W)$ with relative $W$-valued forms:

$$
F_{\delta}\left(\Omega_{\epsilon}^{q}(W)\right) \hookrightarrow \Omega^{q}\left(B_{\delta}(0) \times B_{\epsilon}(0) / B_{\delta}(0), W\right) .
$$

On the other hand, every relative $q$-form is in the image of (25) after shrinking $\delta$ and $\epsilon$ appropriately.

Let $\epsilon^{\prime} \in \mathbb{R}_{+}^{n}$ be a multiradius which is component-wise strictly smaller than $\epsilon$, written $\epsilon^{\prime}<\epsilon$. There is a continuous inclusion $i: \Omega_{\epsilon}^{q}(W) \hookrightarrow \Omega_{\epsilon^{\prime}}^{q}(W)$.

Lemma 26 (Poincaré lemma). Let $\epsilon^{\prime}<\epsilon$ and $q>0$. Then there exists a bounded linear map

$$
h: \Omega_{\epsilon}^{q}(W) \rightarrow \Omega_{\epsilon^{\prime}}^{q-1}(W)
$$

such that $d \circ h+h \circ d=i$.

Proof. We have

$$
\Omega_{\epsilon}^{q}(W)=\bigoplus_{1 \leq k_{1}<\cdots<k_{q} \leq n} F_{\epsilon}(W) d x_{k_{1}} \ldots d x_{k_{q}} .
$$

Set $C:=\max _{i}\left(\epsilon_{i} / \epsilon_{i}^{\prime}\right)$. By assumption we have $C>1$. Hence, for integers $N \gg 0$ we have $|1 /(N+q)| \leq C^{N}$. We define

$$
h\left(x^{I} d x_{k_{1}} \ldots d x_{k_{q}}\right):=\frac{1}{|I|+q} \sum_{\alpha=1}^{q}(-1)^{\alpha-1} x^{I+e_{k_{\alpha}}} d x_{k_{1}} \ldots \widehat{d x_{k_{\alpha}}} \ldots d x_{k_{q}} .
$$

and

$$
h\left(\sum a_{I} x^{I} d x_{k_{1}} \ldots d x_{k_{q}}\right):=\sum a_{I} h\left(x^{I} d x_{k_{1}} \ldots d x_{k_{q}}\right) .
$$


Since

$$
\left\|\frac{a_{I}}{|I|+q}\right\| \epsilon^{\prime I} \leq\left\|a_{I}\right\| C^{|I|} \epsilon^{\prime I} \leq\left\|a_{I}\right\| \epsilon^{I} \quad \text { for }|I| \gg 0,
$$

it follows that the power series $\sum_{I} a_{I} /(|I|+q) x^{I+e_{k \alpha}}$ is $\epsilon^{\prime}$-convergent, whence that $h$ is well-defined, and also that $h$ is a bounded linear operator.

By continuity, it is now enough to check the equality $d h+h d=i$ on monomials $x^{I} d x_{k_{1}} \ldots d x_{k_{q}}$. Relabeling the coordinates, we may moreover assume that $\left(k_{1}, \ldots, k_{q}\right)=(1, \ldots, q)$. We have

$$
\begin{aligned}
d h & \left(x^{I} d x_{1} \ldots d x_{q}\right) \\
= & d\left(\frac{1}{|I|+q} \sum_{\alpha=1}^{q}(-1)^{\alpha-1} x^{I+e_{\alpha}} d x_{1} \ldots \widehat{d x_{\alpha}} \ldots d x_{q}\right) \\
= & \left(\frac{1}{|I|+q} \sum_{\alpha=1}^{q}\left(i_{\alpha}+1\right) x^{I} d x_{1} \ldots d x_{q}\right) \\
& +\frac{1}{|I|+q} \sum_{\alpha=1}^{q} \sum_{\beta=q+1}^{n}(-1)^{\alpha-1}(-1)^{q-1} i_{\beta} x^{I+e_{\alpha}-e_{\beta}} d x_{1} \ldots \widehat{d x_{\alpha}} \ldots d x_{q} d x_{\beta} \\
= & \frac{\left(\sum_{\alpha=1}^{q} i_{\alpha}\right)+q}{|I|+q} x^{I} d x_{1} \ldots d x_{q} \\
& +\frac{1}{|I|+q} \sum_{\alpha=1}^{q} \sum_{\beta=q+1}^{n}(-1)^{\alpha+q} i_{\beta} x^{I+e_{\alpha}-e_{\beta}} d x_{1} \ldots \widehat{d x_{\alpha}} \ldots d x_{q} d x_{\beta}
\end{aligned}
$$

and

$$
\begin{aligned}
h d\left(x^{I} d x_{1} \ldots d x_{q}\right) & h\left((-1)^{q} \sum_{\beta=q+1}^{n} i_{\beta} x^{I-e_{\beta}} d x_{1} \ldots d x_{q} d x_{\beta}\right) \\
= & \frac{(-1)^{q}}{|I|+q} \sum_{\alpha=1}^{q} \sum_{\beta=q+1}^{n}(-1)^{\alpha-1} i_{\beta} x^{I+e_{\alpha}-e_{\beta}} d x_{1} \ldots \widehat{d x_{\alpha}} \ldots d x_{q} d x_{\beta} \\
& +\frac{(-1)^{q}}{|I|+q} \sum_{\beta=q+1}^{n}(-1)^{q} i_{\beta} x^{I} d x_{1} \ldots d x_{q} \\
= & \frac{1}{|I|+q} \sum_{\alpha=1}^{q} \sum_{\beta=q+1}^{n}(-1)^{\alpha+q-1} i_{\beta} x^{I+e_{\alpha}-e_{\beta}} d x_{1} \ldots \widehat{d x_{\alpha}} \ldots d x_{q} d x_{\beta} \\
& +\frac{\left(\sum_{\beta=q+1}^{n} i_{\beta}\right)}{|I|+q} x^{I} d x_{1} \ldots d x_{q}
\end{aligned}
$$


Thus, $(d h+h d)\left(x^{I} d x_{1} \ldots d x_{k}\right)=x^{I} d x_{1} \ldots d x_{k}$. This finishes the proof.

Lemma 27. Let $\Phi: W \rightarrow W^{\prime}$ be a bounded linear map between Banach spaces. It induces a map $\Omega_{\epsilon}^{q}(W) \rightarrow \Omega_{\epsilon}^{q}\left(W^{\prime}\right)$, denoted by the same symbol. For $q>0$ and $\epsilon^{\prime}<\epsilon$, the diagram

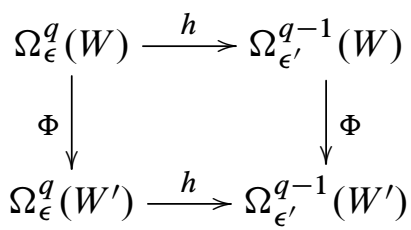

commutes.

Proof. This follows directly from the definitions.

\section{References}

[Féaux de Lacroix 1998] C. T. Féaux de Lacroix, Einige Resultate über die topologischen Darstellungen p-adischer Liegruppen auf unendlich dimensionalen Vektorräumen über einem p-adischen Körper, Ph.D. thesis, Univ. Münster, 1998. pp. 1-111 in Schriftenreihe Math. Inst. Univ. Münster (3) 23, 1999. MR 2000k:22021 Zbl 0963.22009

[Flach 2008] M. Flach, "Cohomology of topological groups with applications to the Weil group", Compos. Math. 144:3 (2008), 633-656. MR 2009f:14033 Zbl 1145.18006

[Huber and Kings 2011] A. Huber and G. Kings, "A p-adic analogue of the Borel regulator and the Bloch-Kato exponential map", J. Inst. Math. Jussieu 10:1 (2011), 149-190. MR 2012a:19010 Zbl 1243.11113

[Huber et al. 2011] A. Huber, G. Kings, and N. Naumann, "Some complements to the Lazard isomorphism”, Compos. Math. 147:1 (2011), 235-262. MR 2012d:22016 Zbl 1268.20051

[Kohlhaase 2011] J. Kohlhaase, “The cohomology of locally analytic representations", J. Reine Angew. Math. 651 (2011), 187-240. MR 2774315 Zbl 1226.22020

[Lazard 1965] M. Lazard, "Groupes analytiques p-adiques”, Inst. Hautes Études Sci. Publ. Math. 26 (1965), 389-603. MR 35 \#188 Zbl 0139.02302

[Lechner 2012] S. Lechner, "A comparison of locally analytic group cohomology and Lie algebra cohomology for $p$-adic Lie groups", preprint, 2012. arXiv 1201.4550

[Schneider 2002] P. Schneider, Nonarchimedean functional analysis, Springer, Berlin, 2002. MR 2003a:46106 Zbl 0998.46044

[Schneider 2011] P. Schneider, p-adic Lie groups, Grundlehren der Mathematischen Wissenschaften 344, Springer, Heidelberg, 2011. MR 2012h:22010 Zbl 1223.22008

[Schneider and Teitelbaum 2002] P. Schneider and J. Teitelbaum, "Locally analytic distributions and p-adic representation theory, with applications to GL 2 ", J. Amer. Math. Soc. 15:2 (2002), 443-468. MR 2003b:11132 Zbl 1028.11071

[SGA $4_{1}$ 1972] M. Artin, A. Grothendieck, and J. L. Verdier (editors), Théorie des topos et cohomologie étale des schémas, Tome 1: Théorie des topos, Exposés I-IV (Séminaire de Géométrie Algébrique du Bois Marie 1963-1964), Lecture Notes in Math. 269, Springer, Berlin, 1972. MR 50 \#7130 Zbl 0234.00007 
Communicated by Marie-France Vignéras

Received 2014-09-09 Revised 2015-02-17 Accepted 2015-04-06

georg.tamme@ur.de

Fakultät für Mathematik, Universität Regensburg, D-93040 Regensburg, Germany 


\section{Algebra \& Number Theory}

msp.org/ant

\section{EDITORS}

MANAGING EDITOR

Bjorn Poonen

Massachusetts Institute of Technology

Cambridge, USA

\author{
EDITORIAL BOARD CHAIR \\ David Eisenbud \\ University of California \\ Berkeley, USA
}

\section{BOARD OF EDITORS}

Georgia Benkart

Dave Benson

Richard E. Borcherds

John H. Coates

J-L. Colliot-Thélène

Brian D. Conrad

Hélène Esnault

Hubert Flenner

Edward Frenkel

Andrew Granville

Joseph Gubeladze

Roger Heath-Brown

Craig Huneke

Kiran S. Kedlaya

János Kollár

Yuri Manin

Barry Mazur

Philippe Michel
University of Wisconsin, Madison, USA

University of Aberdeen, Scotland

University of California, Berkeley, USA

University of Cambridge, UK

CNRS, Université Paris-Sud, France

Stanford University, USA

Freie Universität Berlin, Germany

Ruhr-Universität, Germany

University of California, Berkeley, USA

Université de Montréal, Canada

San Francisco State University, USA

Oxford University, UK

University of Virginia, USA

Univ. of California, San Diego, USA

Princeton University, USA

Northwestern University, USA

Harvard University, USA

École Polytechnique Fédérale de Lausanne
Susan Montgomery

Shigefumi Mori

Raman Parimala

Jonathan Pila

Anand Pillay

Victor Reiner

Peter Sarnak

Joseph H. Silverman

Michael Singer

Vasudevan Srinivas

J. Toby Stafford

Ravi Vakil

Michel van den Bergh

Marie-France Vignéras

Kei-Ichi Watanabe

Efim Zelmanov

Shou-Wu Zhang
University of Southern California, USA

RIMS, Kyoto University, Japan

Emory University, USA

University of Oxford, UK

University of Notre Dame, USA

University of Minnesota, USA

Princeton University, USA

Brown University, USA

North Carolina State University, USA

Tata Inst. of Fund. Research, India

University of Michigan, USA

Stanford University, USA

Hasselt University, Belgium

Université Paris VII, France

Nihon University, Japan

University of California, San Diego, USA

Princeton University, USA

\section{PRODUCTION}

production@msp.org

Silvio Levy, Scientific Editor

See inside back cover or msp.org/ant for submission instructions.

The subscription price for 2015 is US $\$ 255 /$ year for the electronic version, and $\$ 440 /$ year ( $+\$ 55$, if shipping outside the US) for print and electronic. Subscriptions, requests for back issues and changes of subscribers address should be sent to MSP.

Algebra \& Number Theory (ISSN 1944-7833 electronic, 1937-0652 printed) at Mathematical Sciences Publishers, 798 Evans Hall \#3840, c/o University of California, Berkeley, CA 94720-3840 is published continuously online. Periodical rate postage paid at Berkeley, CA 94704, and additional mailing offices.

ANT peer review and production are managed by EditFLOW ${ }^{\circledR}$ from MSP.

\section{PUBLISHED BY}

- mathematical sciences publishers

nonprofit scientific publishing

http://msp.org/

(C) 2015 Mathematical Sciences Publishers 


\section{Algebra \& Number Theory}

Volume $9 \quad$ No. $4 \quad 2015$

Motivic Donaldson-Thomas invariants of small crepant resolutions

ANDREW MORRISON and KENTARO NAGAO

Étale homotopy equivalence of rational points on algebraic varieties

AMBRUS PÁL

Fermat's last theorem over some small real quadratic fields

NUNO FREITAS and SAMIR SIKSEK

Bounded negativity of self-intersection numbers of Shimura curves in Shimura surfaces MARTIN MÖLLER and DOMINGo TOLEDO

Singularities of locally acyclic cluster algebras

Angélica Benito, Greg Muller, Jenna Rajchgot and Karen E. Smith

On an analytic version of Lazard's isomorphism

GEORG TAMME

Towards local-global compatibility for Hilbert modular forms of low weight JAMES NEWTON

Horrocks correspondence on arithmetically Cohen-Macaulay varieties

981

Francesco Malaspina and A. Prabhakar RaO

The Elliott-Halberstam conjecture implies the Vinogradov least quadratic nonresidue conjecture

TERENCE TAO 